\title{
El análisis crítico del discurso como herramienta de investigación psicosocial del mundo del trabajo. Discusiones desde América Latina*
}

\author{
Critical Discourse Analysis as a Tool in Psychosocial Research on
} the World Of Work. Discussions from Latin America

Recibido: abril 8 de 2009 | Revisado: julio 14 de 2009 | Aceptado: julio 20 de 2009

\author{
ANTONIO STECHER ** \\ Universidad Diego Portales, Santiago, Chile
}

El presente artículo fue elaborado en el marco de mi participación en el Programa de Estudios de Doctorado en Psicología Social, del Departamento de Psicología Social, de la Universidad Autónoma de Barcelona. Agradezco a Conicyt (Chile) y a la Fundación Carolina (España) cuyas becas han permitido mi participación y afiliación a dicho programa y departamento. Agradezco, a su vez, los aportes de Lupicinio Iñiguez quien comentó versiones preliminares de este trabajo.

** Universidad Diego Portales (Chile)-Facultad de Psicología. Programa de Estudios Psicosociales del Trabajo. Vergara 275, Santiago-Chile.

\section{RESUMEN}

Este artículo presenta la perspectiva teórico-metodológica del Análisis Crítico del Discurso, y dentro de ésta, el modelo tridimensional de discurso desarrollado por Norman Fairclough. Se destaca el modo en que dichos enfoques pueden enriquecer el campo de la investigación psicosocial sobre el trabajo en América Latina, arrojando luces sobre las dimensiones discursivas de los procesos de reestructuración productiva y flexibilización laboral implementados en diversos países de la región. Se argumenta que a pesar del importante desarrollo y renovación de dicho campo en América Latina en la última década, aún es escasa la incorporación de las herramientas conceptuales del análisis del discurso, lo que ha dificultado el estudio de las emergentes modalidades de uso del lenguaje que caracterizan al nuevo capitalismo.

Palabras clave autor

Análisis Crítico del Discurso, Norman Fairclough, investigación psicosocial, trabajo, América Latina.

Palabras clave descriptor

Fairclough norman, critica e interpretación, análisis del discurso, cambio social.

\section{A B S T R A C T}

This article presents the theoretical-methodological perspective of critical discourse analysis and, within this, a three-dimensional framework of discourse developed by Norman Fairclough. We note the way in which these approaches can enrich the field of psychosocial research on work in Latin America, shedding light on the discursive dimensions of the productive restructuration and work flexibilization processes implemented in diverse countries of the region. We argue that, in spite of the important development and renewal of this field in Latin America over the last ten years, the incorporation of the conceptual tools of discourse analysis remains scarce, thereby hindering the study of the emerging modalities of the use of language that characterize the new capitalism.

Key words author

Critical Discourse Analysis, Norman Fairclough, Psychosocial Research, Work, Latin America.

Key words plus

Fairclough Norman, Criticism and Interpretation, Discourse Analysis, Social Change. 


\section{Introducción}

Uno de los desarrollos más interesantes de la última década dentro de la psicología latinoamericana, es el surgimiento y consolidación de diversos núcleos académicos que han enriquecido con nuevas perspectivas la investigación psicosocial sobre el mundo del trabajo (Braz de Aquino \& Sandoval, 2002; Codo, 2008; Coutinho, Krawulski \& Soares, 2007; Díaz, Godoy, Stecher \& Toro, 2006; Godoy \& Stecher, 2008; Mendes, 2008; Nardi, 2006; Pulido-Martinez, 2004, 2007; Rentería \& Malvezzi, 2008; Román, 2008; Schvarstein \& Leopold, 2005; Sato, 2007; Sisto, 2005; Soto, 2008; Tittoni, 2007; Vargas, Aragón, Rivera \& Trevisi, 2004). Aunque aún claramente marginales respecto a las corrientes profesionales y académicas dominantes de la psicología del trabajo y las organizaciones, estos nuevos desarrollos han dado lugar a una importante producción, fundamentalmente empírica pero también teórica, que ha contribuido significativamente a ampliar la comprensión y reflexión crítica sobre las implicancias psicosociales y sociopolíticas de los procesos de reestructuración productiva y flexibilización laboral implementados en diversos países de la región desde los años 80 (De la Garza, 2000; Stillerman \& Winn, 2006).

Estas diferentes producciones, si bien bastante heterogéneas en términos de sus perspectivas teórico-metodológicas y de sus específicos grados de desarrollo y legitimación en sus contextos institucionales y nacionales, comparten, hasta cierto punto, un conjunto de lineamientos generales. Éstos, si bien no permiten hablar en ningún caso de un programa de investigación homogéneo, articulado e institucionalizado, si dan cuenta de ciertas tendencias de renovación de los estudios psicosociales del trabajo en América Latina. En primer lugar, se trata de aproximaciones interesadas en comprender el trabajo como espacio de construcción de subjetividades, experiencias y significados, y no únicamente como un ámbito de producción económica. Esto implica dejar de lado una mirada objetivante de los trabajadores como recurso a ser gestionado en pos de la maximización de la productividad de la empresa, compren- diéndolos más bien en tanto actores sociales que construyen cotidianamente sentidos individuales y colectivos, y que a través de sus prácticas contribuyen a la producción y transformación de los espacios laborales (Battistini, 2004). Uno de los aportes fundamentales de estas aproximaciones ha sido relevar la heterogeneidad de las subjetividades laborales, visibilizando la enorme complejidad y diferenciación interna del mundo del trabajo (por sectores productivos, por tipo de contrato, por países, etc.), y dando cuenta del modo como los diversos posicionamientos sociales de los sujetos que trabajan (hombre o mujer, viejo o joven, blanco o indígena, etc.) implican experiencias y trayectorias altamente diferenciadas y desiguales. En segundo lugar, se trata de producciones que incorporan una perspectiva sociohistórica a la hora de abordar los entornos y experiencias laborales, destacando, para el caso de las últimas décadas, el modo como la nueva fase y lógica de producción, legitimación y acumulación del capitalismo (global, informacional, flexible) ha implicado una profunda y progresiva reestructuración del mundo (los mundos) del trabajo. Esta perspectiva contextual implica reconocer las articulaciones y codeterminaciones entre los cambios sociolaborales y las transformaciones en otros ámbitos (políticos, económicos, culturales) de la vida social, así como dar cuenta de las muy singulares formas que adquieren los procesos de reestructuración productiva en América Latina, donde coexisten simultáneamente formas productivas prefordistas, fordistas y postfordistas, y donde la flexibilidad laboral ha sido entendida e implementada básicamente en términos de desregulación del mercado laboral y de intensificación y precarización del trabajo (De la Garza, 2000). En tercer lugar, se trata de abordajes que apuestan por complementar o reemplazar las matrices epistemológicas, marcos conceptuales y estrategias metodológicas de raigambre (neo)positivista y comportamentalista de la tradicional psicología del trabajo, por perspectivas hermenéuticas, críticas y/o posestructuralistas que permitan recuperar la subjetividad e historicidad producida e inscrita en los espacios laborales. Así, encontramos que muchas de estas producciones apuestan por la utiliza- 
ción de metodologías cualitativas, buscando romper con modelos empiricistas basados en relaciones entre variables operacionalizadas y cuantificables que han caracterizado los desarrollos hegemónicos de la psicología del siglo XX (Danzinger, 1996; Gergen, 1996). Este desplazamiento ha implicado un cambio de interés desde la búsqueda de leyes universales y abstractas del comportamiento laboral que permitirían controlar y ajustar las respuestas de los sujetos a las demandas de la organización, hacia la exploración de los sentidos, identidades, relatos y prácticas cotidianas que definen las experiencias situadas, múltiples, heterogéneas y contradictorias de los sujetos que trabajan. Experiencias y prácticas condicionadas y determinadas por los contextos sociohistóricos y los marcos organizacionales en que se insertan, pero, al mismo tiempo, capaces de recrear, resistir y transformar, hasta cierto punto, dichas condicionantes. En cuarto lugar, estos desarrollos se caracterizan por asumir, más o menos explícitamente, una sensibilidad crítica que lleva a explorar y cuestionar las diversas formas de explotación, enajenación, sufrimiento, malestar y control que caracterizan la organización del trabajo en las sociedades capitalistas contemporáneas. Dicha perspectiva crítica supone, a su vez, un esfuerzo por visibilizar las prácticas y posibilidades de resistencia de los sujetos que trabajan, así como por construir un conocimiento que contribuya a desnaturalizar y revertir los procesos de precarización, desregulación, desigualdad y mercantilización que son presentados por la retórica neoliberal como apolíticos, naturales e inevitables (Fairclough, 2000a). En quinto lugar, este conjunto de producciones suponen una problematización de la historia, los discursos y los límites de la psicología. Por un lado, se plantea la necesidad de visibilizar el rol constitutivo de los saberes y prácticas psicológicas en la organización (post)industrial del trabajo, el modo en que las prácticas discursivas psi (tanto en la línea taylorista como en la línea de las relaciones humanas) han contribuido a la producción de formas de conocimiento, disciplinamiento y subjetivación laboral funcionales a los requerimientos de la empresa y del capital, o más ampliamente, consustanciales a la operatoria de los diagramas de poder propios de las sociedades modernas (Baritz, 1961; Pulido-Martínez, 2004; Rose, 1996). Por otro lado, se plantea la necesidad de ir más allá de los límites del campo disciplinar, desarrollando proyectos interdisciplinares o transdisciplinares que, reuniendo los aportes de diversas ciencias sociales, permitan abordajes más integrados y complejos del mundo del trabajo. Estos 5 ejes, que en ningún caso constituyen una descripción exhaustiva, permiten dar cuenta de algunas de las características de este emergente territorio de nuevos abordajes psicosociales del mundo laboral, dentro de la psicología latinoamericana

Uno de los aspectos llamativos de estos recientes desarrollos es la relativamente escasa utilización de los recursos teórico-metodológicos del ámbito del análisis del discurso. Salvo algunas excepciones (García \& Carvajal, 2007; PulidoMartínez, 2004, 2007; Pulido \& Carvajal, 2001; Román, 2008; Sisto, 2005; Vargas, Aragón, Rivera $\&$ Trevisi, 2004), y al igual que en el caso de los actuales desarrollos de la sociología del trabajo en América Latina (Antunes, 2001; Battistini, 2004; De la Garza, 2000; Todaro \& Yáñez, 2004), lo que predomina a nivel de los diseños cualitativos de investigación es la lógica del análisis (cualitativo) de contenido, en que se busca extraer a través de procesos de codificación sucesiva (habitualmente inspirados en la Grounded Theory) los principales temas o significados de un texto, el que, más que como un tópico en sí mismo, se ve como un recurso para acceder a ciertos aspectos de la realidad social (Potter \& Wetherell, 1987). Prevalece, así, en muchos de estos estudios, una consideración del lenguaje como mecanismo de representación o expresión de la realidad objetiva, social o subjetiva, prestándose menos atención al discurso en tanto forma de acción y práctica social que tiene un rol constitutivo en los procesos de construcción del conocimiento, regulación de las relaciones sociales y configuración de las identidades (Fairclough, 1992; Jaworski \& Coupland, 1999; Iñiguez, 2003; Martín, 2001; Parker, 1992; Wetherell, 2001). Como señalan Iñiguez y Antaki (1994 en Iñiguez, 2003) hablar de discurso es hablar de "un conjunto de prácticas lingüísticas que mantienen y 
promueven ciertas relaciones sociales. El análisis consiste en estudiar cómo esas prácticas actúan en el presente manteniendo y promoviendo esas relaciones: es sacar a la luz el poder del lenguaje como una práctica constituyente y regulativa" (p. 99).

Esta escasa y poco sistemática utilización de las herramientas conceptuales y analíticas del campo del análisis del discurso en los Estudios Sociales del Trabajo en América Latina ${ }^{1}$-algo que contrasta por ejemplo con la fuerte presencia de diversas perspectivas discursivas en los estudios organizacionales del mundo anglosajón (Alvesson \& Karreman, 2000; Alvesson \& Willmott, 2002; Du Gay, 1996; Fairclough, 2005; Grant, Keenoy \& Oswick, 1998; Knights \& Morgan, 1991; Mumby $\&$ Clair, 2000)-constituye una debilidad no menor si consideramos que los cambios en el mundo del trabajo, producidos en las últimas décadas, están asociados a importantes cambios y rearticulaciones a nivel del discurso (Fairclough, 1992, 1995, 2002). Es decir, han implicado el despliegue y la articulación discursiva de nuevas representaciones e imaginarios sobre el trabajo, la empresa y la economía (trabajo flexible, discurso managerial, cultura empresarial, discurso neoliberal, etc.); de nuevas formas de interacción comunicativa entre los trabajadores, y entre éstos y las jefaturas y los clientes (trabajo en equipo, entrevistas de evaluación individualizada de la productividad, trato cordial y estandarizado a clientes, etc.); y de nuevas identidades sociales y posiciones subjetivas caracterizadas por particulares maneras de hablar y usar el lenguaje (sujeto emprendedor, manager, etc.) (Adkins \& Lury, 1999; Bauman, 2000; Boltanski \& Chiapello, 2002; Bourdieu, 2000; Hard \& Negri, 2002; Sennet, 2006).

A la luz de lo anterior, es posible plantear que una de las tareas y desafíos de las nuevas perspectivas de investigación psicosocial sobre el mundo del trabajo en América Latina, es incorporar

1 Por Estudios Sociales del Trabajo en América Latina nos referimos en general a la producción global de las ciencias sociales de la región sobre la temática laboral. Dentro de ese campo, y como un subcampo específico, ubicamos a los estudios psicosociales del trabajo, los cuales están vinculados fundamentalmente a la Psicología Social y a la Psicología del Trabajo y las Organizaciones. dentro de su arsenal conceptual y metodológico los recursos desarrollados dentro del campo de los Estudios Contemporáneos del Discurso (Van Dijk, 2000), de modo de poder visibilizar teóricamente, indagar empíricamente y discutir críticamente las emergentes modalidades de uso del lenguaje que caracterizan al nuevo capitalismo y, más específicamente, a las recientes transformaciones del mundo del trabajo en la región.

El presente artículo busca contribuir en esa dirección a partir de la presentación de la perspectiva teórica del Análisis Crítico del Discurso (ACD), centrándose dentro de ésta en el modelo tridimensional de discurso desarrollado por el lingüista británico Norman Fairclough. A lo largo de la argumentación buscaremos destacar e ilustrar el modo como el ACD en general, y el enfoque de Fairclough en particular, ofrecen valiosas herramientas para explorar las dimensiones discursivas del mundo laboral. Además, como veremos, dichas herramientas resultan en general consistentes con las características que hemos destacado como distintivas de las nuevas perspectivas de investigación psicosocial sobre el mundo del trabajo, que se vienen desarrollando en América Latina.

\section{El análisis crítico del discurso (ACD)}

El ACD es una de las diversas perspectivas teóricometodológicas que podemos identificar al interior del campo contemporáneo de los Estudios del Discurso $^{2}$ (Iñiguez, 2003; Wetherell, 2001). Se trata de un campo académico heterogéneo e interdisciplinar que empieza a gestarse en forma paralela en diferentes disciplinas desde mediados de los años 60 , que adquiere una progresiva institucionalización (revistas, congresos, cursos de formación, núcleos de investigación, manuales, etc.) a partir de los años 80 , y cuyo principal interés es el estudio

2 Van Dijk (2003a) propone la nominación de "Estudios Contemporáneos del Discurso", en vez de la generalmente utilizada "Análisis del discurso", para dar cuenta de que el campo "No sólo incluye "análisis", sino también "teorías", "aplicaciones", "crítica” y otras dimensiones de investigación...” (p. 12). En este trabajo usamos indistintamente ambas denominaciones. 
empírico de los usos del lenguaje, hablado o escrito, en sus contextos (macro y/o micro) sociales específicos (Calsamiglia \& Tusón, 1999; Iñiguez, 2003; Taylor, 2001; Thompson, 1984; Wetherell, 2001). El surgimiento y consolidación de este campo de los estudios contemporáneos del discurso -donde convergen los aportes de diferentes disciplinas tales como la lingüística, la antropología, la psicología, la sociología, la filosofía, las ciencias de la comunicación (Iñiguez, 2003; Van Dijk, 2003a) - es una expresión más del creciente interés de la ciencias sociales y humanas por discutir e investigar el lugar del lenguaje en la vida social, destacando su carácter de acción y práctica social, así como sus efectos productivos y constitutivos a nivel de los saberes, las interacciones y las subjetividades que definen la especificidad de un particular ordenamiento sociohistórico (Jaworski \& Coupland, 1999; Iñiguez, 2003; Martín, 2001).

Es dentro de este amplio y heterogéneo ${ }^{3}$ campo de los Estudios del Discurso —el cual, por cierto, es una de las expresiones del desarrollo a lo largo del siglo XX, primero en la filosofía y posteriormente en las Ciencias Sociales, de lo que se ha venido a denominar el "giro lingüístico" (Rorty, 1990)_ donde debemos situar la perspectiva del Análisis Crítico del Discurso (ACD).

El ACD tiene como antecedente directo e inmediato los desarrollos de la denominada Lingüística Crítica, la que desde los años 70 en Gran Bretaña se interesó por estudiar, con herramientas analíticas propiamente lingüísticas, las relaciones entre lenguaje, poder y control social (Fowler, Kress, Hodge \& Trew, 1979). En términos más amplios el ACD tiene sus raíces y se nutre del desarrollo del denominado marxismo occidental, el

3 Se trata de un campo donde coexisten enfoques muy diversos en términos de sus filiaciones disciplinares, sus ámbitos de investigación, los tipos de datos privilegiados, las perspectivas lingüísticas en que se sustentan, así como de los presupuestos ontológicos, epistemológicos y ético-políticos que orientan la investigación (Iñiguez, 2003; Martín, 2001; Van Dijk, 2000; Wetherell, 2001). Algunos de los enfoques más importantes desde la perspectiva de las ciencias sociales son: La Sociolingüística interaccional, la Etnografía de la comunicación, el Análisis de la conversación, la Psicología (social) discursiva, las Aproximaciones foucultianas, las Perspectivas Bakhtinianas y el Análisis crítico del discurso (Iñiguez, 2003; Jaworski \& Coupland, 1999; Wetherell, 2001). que a lo largo del siglo XX - de Gramsci al marxismo cultural inglés, pasando por los trabajos de las distintas generaciones de la Escuela de Frankfurt y de Althusser-destacó la centralidad de la cultura y las formas simbólicas en los procesos de dominación característicos de las sociedades capitalistas, así como en las posibilidades de subvertir y resistir dichas relaciones de poder (Fairclough, 1992; Fairclough \& Wodak, 2000). A su vez, los trabajos del círculo de Bakhtin, así como algunas de las reflexiones de Foucault sobre el orden del discurso y las formaciones discursivas son también parte de los orígenes teóricos del ACD (Fairclough \& Wodak, 2000).

Sin embargo, como red y campo de investigación articulado y relativamente institucionalizado en torno a la etiqueta de ACD, la perspectiva del Análisis Crítico del Discurso es más bien reciente. Como señala Ruth Wodak (2003), es a inicios de los años 90 cuando surge el ACD como tal, a partir de un simposio realizado en Amsterdan en que participaron Teun Van Dijk, Norman Fairclough, Gunther Kress, Theo Van Leeuwen y Ruth Wodak, los que sin duda pueden ser considerados como los fundadores y los autores más importantes y reconocidos dentro del campo del ACD. ${ }^{4}$ Hoy en día, el ACD es una perspectiva consolidada y legitimada dentro del campo de los Estudios del Discurso, y es posiblemente el enfoque de Análisis del Discurso que más desarrollo ha tenido en América Latina (Berardi, 2003; Bolívar, 2004; Magalhaes, 2005; Morales \& Cortez, 2005; Pardo \& Buscaglia, 2008) gracias, en gran medida, a la presencia permanente de Van Dijk en distintos países de la región (Martín \& Whittaker, 1998).

Algunos de los principales postulados teóricos del ACD son los siguientes: en primer lugar, se caracteriza por desarrollar una teorización que releva la relación dialéctica entre el discurso y las

4 Hay que señalar que, si bien en esta sección el foco de nuestro argumento está puesto en aquellos elementos compartidos dentro del ACD, no es menos cierto que dentro de dicho campo existen distintos sub-enfoques con sus particularidades teóricas y metodológicas, siendo quizás los más destacados el enfoque cognitivo de Van Dijk, el método histórico-discursivo de Wodak, la perspectiva semiótica social de Kress y Van Leeuwen y la perspectiva de cambio sociocultural y cambio discursivo de Fairclough (Martín \& Whittaker, 1998). 
estructuras sociales, dando cuenta del modo como el discurso, en tanto que práctica social históricamente situada, es, por un lado, modelado y determinado por las macro y micro estructuras en las que está inserto, al mismo tiempo que, por otro lado, contribuye activa y creativamente a la construcción, sostenimiento y transformación del orden social (Fairclough \& Wodak, 2000). En segundo lugar, el ACD se caracteriza por plantear que el discurso es una de las dimensiones de la vida social, pero que ésta última, en ningún caso, puede reducirse al lenguaje o más en general a la semiosis. Lo discursivo es un momento de la vida social que incide en los otros momentos o elementos no discursivos, al mismo tiempo que es modelado por estos últimos (Chouliaraki \& Fairclough, 1999; Van Dijk, 2003b; Wetherell, 2001). El desafío teórico es, en este punto, "evitar el error de sobreenfatizar tanto la social determinación del discurso, por un lado, como la construcción de lo social en el discurso, por otro lado. Lo primero convierte al discurso en un mero reflejo de una realidad social más profunda, lo segundo cae en un idealismo que piensa al discurso como la fuente única de lo social" (Fairclough, 1992, p. 65). En tercer lugar, y en lo que es quizás la característica más conocida del ACD, éste se caracteriza por estudiar especialmente las relaciones entre lenguaje y poder, buscando relevar el modo en que el discurso juega un rol central en los mecanismos de dominación y control social característicos de las sociedades capitalistas contemporáneas (Fairclough \& Wodak, 2000; Van Dijk, 1993). Como escribe Wodak (2003), el objetivo central del ACD es estudiar "ya sean éstas opacas o transparentes, las relaciones de dominación, discriminación, poder y control, tal como se manifiestan a través del lenguaje. En otras palabras, el ACD se propone investigar de forma crítica la desigualdad social tal como viene expresada, señalada, constituida, legitimada, etc., por los usos del lenguaje (es decir en el discurso)" (p. 19); o en palabras de Van Dijk (2003b), el foco del ACD es "el estudio crítico de la reproducción discursiva de la dominación en la sociedad" (p. 151). En cuarto lugar, y estrechamente vinculado a lo anterior, el ACD se caracteriza por un espíritu crítico, lo que supone un compromiso explícito con los sujetos sociales más desfavorecidos, así como un interés por generar formas de conocimiento que contribuyan a la democratización y emancipación de la sociedad. Se trata, así, en la tradición de la teoría crítica (Leyva, 2005), de generar un saber que más que limitarse a constatar lo dado y reificar el orden existente, sea capaz de visibilizar las (legitimadas) injusticias (opacadas) contradicciones y (naturalizadas) desigualdades del presente, abriendo así nuevas posibilidades de autocomprensión y acción social para los sujetos sociales (Habermas, 1990; Martín, 2001). Este espíritu crítico es lo que está en la base del interés del ACD por estudiar, no sólo las formas en que el discurso contribuye a sostener las relaciones de dominación, sino también el modo en que a través del discurso se abren posibilidades de resistencia (contradiscursos, discursos alternativos) ante los marcos culturales hegemónicos. En quinto y último lugar, es posible señalar que el ACD posee una identidad disciplinar caracterizada por la mixtura de un poderoso anclaje en la tradición lingüística, por un lado, con una muy fuerte vocación transdiciplinar, por otro lado. La mayoría de los principales exponentes del ACD provienen del campo de los estudios del lenguaje, y hay un fuerte y minucioso uso de los recursos analíticos de la lingüística en esta perspectiva del Análisis del Discurso. Como diría Fairclough (1992), se trata de un análisis del discurso con una potente orientación textual y lingüística. Sin embargo, junto a este fuerte arraigo en las teorías y las estrategias analíticas de la lingüística, el ACD se caracteriza por una importante vocación transdisciplinar, pues entiende que abordar las complejas relaciones entre lenguaje y sociedad requiere el aporte de diversos saberes y teorías. Uno podría entender el ACD como el esfuerzo de articular en una perspectiva de investigación multidisciplinar los recursos teóricoanalíticos de la lingüística, junto a los diversos desarrollos de la teoría social que a lo largo del siglo $\mathrm{XX}$ han buscado problematizar críticamente las diversas articulaciones entre la cultura, el lenguaje y la dominación. Como escribe Van Dijk (2003b) "el ACD debería ser esencialmente diverso y multidisciplinar. (...) El ACD puede realizarse en, o 
combinarse con, cualquier enfoque y subdisciplina de las humanidades y las ciencias sociales" (p. 144).

De esta breve caracterización del ACD podemos destacar tres aspectos que dan cuenta de la fecundidad de esta perspectiva para los nuevos estudios psicosociales del trabajo. Por un lado, se trata de un enfoque que, si bien dedicado al análisis del discurso, no reduce la dinámica de la vida social a sus componentes discursivos o a sus microeventos conversacionales, como sucede, respectivamente, con ciertas perspectivas post- estructuralistas o etnometodológicas dentro de la teoría social en general, o al interior del campo del análisis del discurso, en particular. Esto es particularmente relevante para el campo de los Estudios Sociales del Trabajo en América Latina, donde en ocasiones se identifica el campo del análisis del discurso con un enfoque predominantemente discursivo o microinteraccional de los procesos sociales, y luego, hecha esa asociación, se concluye que dicho estilo de investigación no es pertinente para las temáticas sociolaborales, debido a que no permite abordar adecuadamente las ineludibles dimensiones materiales y socioestructurales del mundo del trabajo. Por otro lado, se trata de una perspectiva que permite indagar y problematizar el modo como los discursos juegan un rol central en el sostenimiento de las relaciones de dominación que caracterizan los espacios laborales, lo que resulta afín al ímpetu crítico que caracteriza las nuevas líneas de investigación psicosocial que venimos comentando. El ACD, así, puede ser un importante aporte a la hora de iluminar las distintas modalidades en que el lenguaje es utilizado en los contextos de trabajo para producir particulares representaciones, formas de acción e identidades que contribuyen a sostener relaciones de control y dominación de ciertos grupos sobre otros. Por último, se trata de un enfoque especialmente interesado y abierto al desarrollo de formas de trabajo interdisciplinar, pero que, al mismo tiempo, hace un uso riguroso de las herramientas analíticas de la lingüística, las que resultan fundamentales para llevar a cabo el tipo de análisis -funcionalmente orientado- del lenguaje que caracteriza el campo de los Estudios del discurso ${ }^{5}$ (Jaworski \& Coupland, 1999). Como hemos señalado, los nuevos núcleos de investigación psicosocial del trabajo en América Latina, han tendido a priorizar un tipo de abordaje que se centra en los contenidos de los textos analizados, prestando menos atención a las funciones que éstos cumplen en el marco de los contextos de interacción específicos en que se han producido. El ACD, así, puede ser un importante aporte en la línea de explorar el modo en que el lenguaje es movilizado en los espacios laborales como un recurso para la acción, esto es, la manera en que las personas, grupos e instituciones persiguen en el trabajo ciertos propósitos y efectúan ciertas acciones a través de las prácticas lingüística (Iñiguez, 2003).

En la siguiente sección, y partir de la presentación del modelo tridimensional del discurso desarrollada por Fairclough, buscaremos precisar y profundizar en las herramientas conceptuales que ofrece la perspectiva del ACD, así como en la utilidad de las mismas para la investigación psicosocial sobre el mundo del trabajo.

\section{Norman Fairclough y el modelo tridimensional de discurso}

Dentro de la perspectiva general del ACD, el sello característico del trabajo de Fairclough es su interés por desarrollar una teoría social crítica del discurso que, a partir de la articulación de los estudios del lenguaje y los desarrollos de las ciencias sociales, oriente la investigación empírica sobre los cambios contemporáneos en el discurso, entendidos como

5 Uno de los aspectos distintivos del análisis del discurso respecto a otras formas de investigación cualitativa es, justamente, la detallada atención que presta a las propiedades lingüísticas (en un sentido amplio) del texto. Así, hacer análisis de discurso exige analizar "algunas de las detalladas estructuras, estrategias y funciones del texto y la conversación, lo que incluye las formas -gramaticales, pragmáticas, de interacción, estilísticas, retóricas, semióticas, narrativas o similares- de la organización verbal y paraverbal de los acontecimientos comunicativos" (Van Dijk, 2003b, p. 146). Es sólo a través de este minucioso análisis del texto que es posible proponer y justificar una interpretación respecto a las funciones y a los efectos discursivos que un determinado texto tiene en un contexto micro o macro social específico (Antaki, Billig, Edwards \& Potter, 2003; Iñiguez, 2003). 
parte de procesos más amplios de transformación sociocultural (Chouliaraki \& Fairclough, 1999; Fairclough, 1992). Su tesis central es que no podemos comprender adecuadamente los procesos de reconfiguración societal de las últimas décadas (modernidad tardía), si no atendemos a las modificaciones en las prácticas discursivas en que dichos procesos de cambio se expresan, al mismo tiempo que se sostienen. A su vez, no lograremos comprender los discursos que actualmente se producen en los distintos ámbitos de la vida social, si no los analizamos desde la perspectiva y matriz más amplia del cambio sociocultural (Fairclough, 1995). Esta perspectiva resulta de particular interés para los nuevos desarrollos de investigación psicosocial del trabajo en América Latina, en la medida en que éstos se han centrado prioritariamente en comprender las características y especificidades nacionales y regionales de los procesos de reestruccturación productiva y flexibilización laboral que han transformando, en las últimas décadas, los entornos y las experiencias de trabajo. Así, como esperamos mostrar a continuación, el modelo conceptual desarrollado por Fairclough puede ser de gran utilidad para indagar en las dimensiones discursivas de los recientes cambios sociolaborales, en el modo como el trabajo en nuestros países (periféricos y semi-industrializados) bajo el marco del nuevo capitalismo, supone, se expresa y se sostiene en particulares y emergentes prácticas discursivas.

Fairclough (1995) va a definir el discurso como el lenguaje en uso considerado como una forma de práctica social, y al análisis del discurso como el análisis de cómo los textos (hablados o escritos) operan dentro de una práctica sociocultural. Al alero de las características generales del ACD ya mencionadas, y de su particular interés por estudiar el cambio social en las sociedades contemporáneas, Fairclough va a proponer un modelo teórico de tres dimensiones para pensar el discurso y el análisis del discurso. ${ }^{6}$ Este modelo tridimensional consis-

6 En sus últimos trabajos Fairclough (2001, 2003a, 2003b, 2005) ha complejizado este modelo a partir de una comprensión del discurso como categoría que designa los elementos semióticos de la vida social, la que debe ser entendida como redes de prácticas sociales compuestas de momentos discursivos y no discursivos. te en conceptualizar y analizar cualquier "evento discursivo" en términos de tres facetas o dimensiones: (i) como un texto (pieza de lenguaje escrito o hablado), (ii) como un caso de práctica discursiva (discursive practice) que involucra los procesos de producción e interpretación de textos, y como (iii) parte de una práctica social, en términos del marco situacional e institucional del evento discursivo.

\section{El discurso como texto}

El texto es la pieza de lenguaje escrito o hablado que constituye el material empírico del investigador, y que es el resultado de un proceso más amplio de producción de textos. A modo de ejemplo, un texto es el discurso del gerente general de una empresa en la junta de accionistas, o el documento que contiene la política pública de un Estado sobre generación de empleos, o el contenido que aparece en la página web de una cadena de supermercados, o el manual de un curso de capacitación de atención al cliente, o una conversación entre una cajera y un cliente, o entre un empleado y su supervisor. Cada uno de esos textos supone una particular y situada utilización del lenguaje donde se actualiza el poder constructivo del discurso. Siguiendo la aproximación funcional al lenguaje de Halliday (1994), Fairclough $(1992,1995)$ va a distinguir tres aspectos de esta potencia constitutiva del discurso que se manifiesta en todo texto: por un lado, el discurso contribuye a modelar las relaciones sociales entre las personas, en la medida en que muchas formas de interacción (por ejemplo, la relación entre un jefe y su subordinado) se definen en buena medida en función de las particulares formas de comunicarse entre sí de los participantes de dicha relación. En segundo lugar, el discurso produce y pone en circulación particulares representaciones y creencias acerca de la realidad. En tercer lugar, el discurso contribuye a la construcción de identidades sociales al ubicar a los sujetos que interactúan discursivamente en ciertas posiciones que suponen particulares formas de ser y estar en el mundo. Así, todo texto, en tanto lenguaje en uso, contribuye simultáneamente a la constitución de (i) acciones/ 
relaciones sociales, (ii) sistemas de creencias y conocimientos, e (iii) identidades sociales.

En este primer nivel, el análisis del discurso supone llevar a cabo un análisis lingüístico detallado del texto (o los textos) dando cuenta del modo como sus distintos rasgos (gramaticales, fonológicos, de vocabulario, de estructura global, de cohesión) producen efectos de sentido en los tres planos mencionados: (inter)acción, representación, identificación (Fairclough, 2003a).

\section{El discurso como práctica discursiva}

Esta dimensión da cuenta del hecho de que todo evento discursivo debe ser pensado en términos de los procesos de producción que le han dado lugar, así como de los procesos de recepción/interpretación a los que se verá sometido en la trama social. El foco de atención, acá, es el modo en que los sujetos producen e interpretan ${ }^{7}$ los textos a partir de un conjunto de recursos discursivos socialmente disponibles y relativamente estables que constituyen órdenes del discurso. Un orden del discurso es entendido como "un set ordenado de prácticas discursivas asociadas con un particular dominio social o institución (entrevista de trabajo, consulta médica, clase escolar, conversación informal)" (Fairclough, 1995, p. 12). Se trata de particulares formas de usar el lenguaje para interactuar, representar e identificarse en un dominio social específico. ${ }^{8}$ Ade-

7 Si bien la noción de práctica discursiva atiende a los procesos de producción e interpretación, en general el foco del análisis y la argumentación de Fairclough, como suele suceder en el campo de los Estudios del Discurso, está centrada en los procesos de producción. En atención a eso, en lo que sigue, nos focalizaremos en esa dimensión.

8 Las prácticas discursivas que componen un orden del discurso pueden ser clasificadas en tres tipos: (i) géneros, (ii) discursos y (iii) estilos (Fairclough, 2003b). Los géneros son "formas socialmente ratificadas de utilizar el lenguaje en conexión con un particular tipo de actividad social" (Fairclough, 1995, p. 14). Dan cuenta del uso del lenguaje para el desarrollo y la coordinación de acciones. Así por ejemplo, la práctica social de trabajar como cajera en un supermercado supone un tipo de actividad que se caracteriza, entre otros aspectos, por interactuar discursivamente con los clientes de un modo regular y estabilizado (saludar, preguntar, ofrecer, despedirse, etc.). Los discursos son formas estabilizadas de representar ciertos aspectos del mundo social, dan cuenta del uso del lenguaje para configurar conocimientos y creencias acerca de la realidad. Así, por ejemplo, las cajeras en un supermercado participan en la producción de representaciones, conocimientos más de los órdenes del discurso de carácter local, es posible hablar, a un nivel más general, de un orden del discurso de la sociedad, el que da cuenta de las relaciones y límites entre los órdenes locales del discurso. Los órdenes del discurso son la faceta discursiva del orden social, y sus componentes, sus límites, así como sus relaciones internas (entre las distintas prácticas discursivas que lo componen) y externas (entre diferentes órdenes locales del discurso) se modifican al compás de los cambios a nivel de los procesos sociohistóricos en los que están insertos (Chiapello \& Fairclough, 2002). Es importante señalar que no existe una relación lineal y mecánica entre un orden del discurso y los textos producidos al interior del dominio social específico del que éste es parte. Este nivel del modelo teórico apunta justamente a dar cuenta de cómo los sujetos utilizan los recursos de un orden del discurso para configurar sus textos, y cómo en ese proceso, al mismo tiempo que reproducen dichos patrones de prácticas discursivas, son capaces de articular y recrear creativamente los mismos (Fairclough, 1995). En parte, esto es posible debido al hecho de que un mismo orden del discurso contiene elementos diversos, complementarios y en ocasiones antagónicos, y a que los sujetos pueden, hasta cierto punto, apelar a recursos discursivos de diferentes ámbitos (órdenes del discurso) en su

y opiniones sobre distintos ámbitos de la vida social (el consumo de los clientes, las dificultades de articular vida familiar y laboral, la dirección de la empresa, etc.) y sobre el empleo de cajera en particular (definiciones de ser cajera, valoraciones de ese empleo, comparaciones con otros puestos de un supermercado, etc.). Es importante distinguir este uso específico de la noción de discurso en tanto representación, del "discurso" como categoría abstracta general que alude al lenguaje en uso. Los estilos son formas relativamente estabilizadas de ser y actuar discursivamente, y dan cuenta del hecho que los sujetos al participar de una interacción discursiva son posicionados de particulares maneras dentro del campo de relaciones sociales, adquiriendo ciertas identidades que dependen, hasta cierto punto, del particular modo de hablar, escribir, comunicarse. Así, por ejemplo, el negocio del supermercado posiciona a particulares actores en la posición de clientes y cajeras, las que son identidades sociales que ocupan los sujetos y que se caracterizan (junto a otros elementos) por una particular manera de utilizar el lenguaje (hablar primero o hablar después, utilizar el lenguaje para preguntar/demandar o para responder, utilizar un tono alto o un tono bajo de voz, etc.). Cada campo social se caracteriza por un particular orden del discurso, esto es, por una particular articulación de géneros (formas de actuar), discursos (formas de representar y conocer) y estilos (formas de ser) (Fairclough 2003a, 2003b). 
praxis social. Dicho en otros términos, los sujetos sólo pueden producir textos a partir de recurrir a los sets de convenciones (orden del discurso) que tienen disponibles y que están socialmente legitimados para ámbitos de acción específicos, pero en ese uso concreto y situado del lenguaje, y producto del carácter abierto de todo evento, de la capacidad reflexiva de la agencia humana y de la heterogeneidad (inter e intra) de los órdenes del discurso, hay lugar para ciertos niveles de creación e innovación. Este segundo nivel referido a las prácticas discursivas y al orden del discurso, juega un rol central en el modelo tridimensional de Fairclough. Es este el nivel que permite establecer una mediación entre la estructura social y los textos como acontecimientos singulares. La estructura y las relaciones sociales dominantes en un particular momento histórico, dan lugar a la estabilización de particulares órdenes del discurso, los que condicionan en los distintos dominios sociales los procesos de producción (e interpretación) de los textos por parte de los actores sociales. De esta manera, es posible dar cuenta del proceso de determinación de la estructura social sobre el uso del lenguaje a partir de los órdenes del discurso. Pero al mismo tiempo, y en la dirección opuesta, los sujetos pueden bajo ciertas condiciones hacer usos creativos e innovadores de los recursos disponibles en uno o varios órdenes del discurso, de ese modo incidir y modificar ciertos aspectos de los órdenes del discurso, y a través de ello generar cambios en la estructura social.

Esta segunda dimensión del discurso se corresponde, en términos de análisis, con el análisis intertextual. Éste supone realizar un detallado análisis interdiscursivo buscando dar cuenta, justamente, de cómo un determinado texto es el producto de la mezcla y combinación de distintos recursos discursivos (géneros, discursos, estilos) estabilizados en órdenes del discurso. ${ }^{9}$ La tarea del analista es

9 Este nivel del análisis intertextual incluye, además del análisis interdiscursivo o de intertextualidad constitutiva, el análisis de intertextualidad manifiesta, el que busca dar cuenta del modo en que en un texto particular aparecen otros textos concretos con los que se dialoga, a modo de citas o reportes, o en forma de presuposiciones (Fairclough, 1992, 2003a). comprender el modo en que un texto particular ha movilizado específicos discursos, géneros y estilos y los ha articulado de un modo singular en sus características semánticas, gramaticales y lexicales, produciendo de ese modo particulares efectos de sentido a nivel de los tres planos ya aludidos: (inter)acción, representación e identificación. ${ }^{10}$

\section{El discurso como práctica social}

Esta tercera dimensión da cuenta de que todo evento discursivo es parte de una práctica social (trabajar, enseñar, etc.) y está, como tal, inserto en un conjunto de situaciones, instituciones y macrocontextos que condicionan fuertemente los usos del lenguaje. Dichas condicionantes deben considerarse en diferentes niveles de abstracción: el nivel concreto de la situación social en que el evento discursivo ocurre (por ejemplo, la interacción entre una cajera y un cliente en un supermercado), el nivel institucional del cual la situación social es parte (por ejemplo, trabajo en el sector comercio), y el nivel más amplio de la sociedad como un entramado de diversas estructuras políticas, económicas y culturales (por ejemplo, capitalismo global). Esta tercera dimensión del modelo busca destacar que todo evento discursivo tiene efectos políticos e ideológicos, en la medida en que es parte constitutiva de prácticas sociales que cotidianamente producen y sostienen (o transforman) las relaciones de dominación imperantes en una sociedad. La tesis de Fairclough, quien sigue en este punto las reflexiones de Gramsci, es que el poder de un grupo social se basa, en gran medida, en el establecimiento de una hegemonía cultural, lo cual supone la regulación de los discursos en distintos dominios de la vida social. El control de los discursos se logra a través de la estabilización de particulares órdenes del discurso a los que los

10 El plano de la interacción, la representación y la identificación a nivel del texto, se corresponden, respectivamente, con los géneros, discursos y estilos a nivel del orden del discurso. Así, por ejemplo, es a través de la movilización y articulación de ciertos estilos que un texto produce efectos de sentido a nivel de las identidades sociales de los participantes en una interacción discursiva. Esta misma lógica se da para el caso de la interacción y los géneros, y la representación y los discursos (Fairclough, 2003a). 
sujetos no pueden sino acudir para actuar (discursivamente) en la vida social. Al apelar a esos órdenes del discurso inscritos en el sentido común, los sujetos movilizan, reproducen y naturalizan aquellas formas de acción, representación e identificación que son funcionales a los grupos dominantes, y de este modo participan del proceso de reproducción ideológica de la sociedad (Fairclough, 1989, 1992, 2000b). Sin embargo, no hay que olvidar que el poder y la hegemonía son siempre estabilizaciones provisorias en el marco de diversas luchas sociales, estabilizaciones que pueden ser impugnadas, resistidas e incluso transformadas a partir, entre otros aspectos, de un cambio en los órdenes del discurso de los distintos dominios de la vida social (Fairclough, 1989, 1992, 1995).

Esta tercera dimensión del discurso se corresponde, a nivel del análisis, con el desarrollo de un análisis social explicativo que busca dar cuenta de las condicionantes sociocontextuales de los eventos discursivos bajo estudio, así como del modo en que éstos -a partir de la movilización de particulares formas de interacción, representaciones e identidades- participan de los procesos de producción y sostenimiento (o transformación) de las formas de dominación imperantes en un particular dominio social. Este nivel del análisis exige "ver al discurso como parte de procesos de lucha social, dentro de una matriz de relaciones de poder" (Fairclough, 1989, p. 163).

Este modelo tridimensional de discurso propuesto por Fairclough -que es tanto un marco conceptual como un modelo de análisis- puede ser un aporte importante en el esfuerzo por indagar, desde una perspectiva crítica, el lugar del discurso en los procesos de cambio sociolaboral en América Latina, permitiendo una mejor comprensión de la nuevas hegemonías, formas de dominación y procesos de resistencia que caracterizan el mundo del trabajo en la región. La Figura 1 sintetiza este modelo (Fairclough, 1989, 1992, 1995).

Este modelo conceptual y de análisis del discurso permite formular y orientar la investigación psicosocial empírica en torno a un conjunto de interrogantes tales como: ¿Cuáles son aquellas prácticas discursivas, aquellos cambios en los usos del lenguaje, en que se expresan y sostienen los procesos de flexibilización laboral y reestructuración productiva ocurridos en las últimas décadas en América Latina? ¿Qué nuevas representaciones del trabajo, la sociedad y los trabajadores, y qué nuevas modalidades de acción e identificación laboral vehiculizan los diversos textos (orales, escritos) que cotidianamente producen empresas, trabajadores,

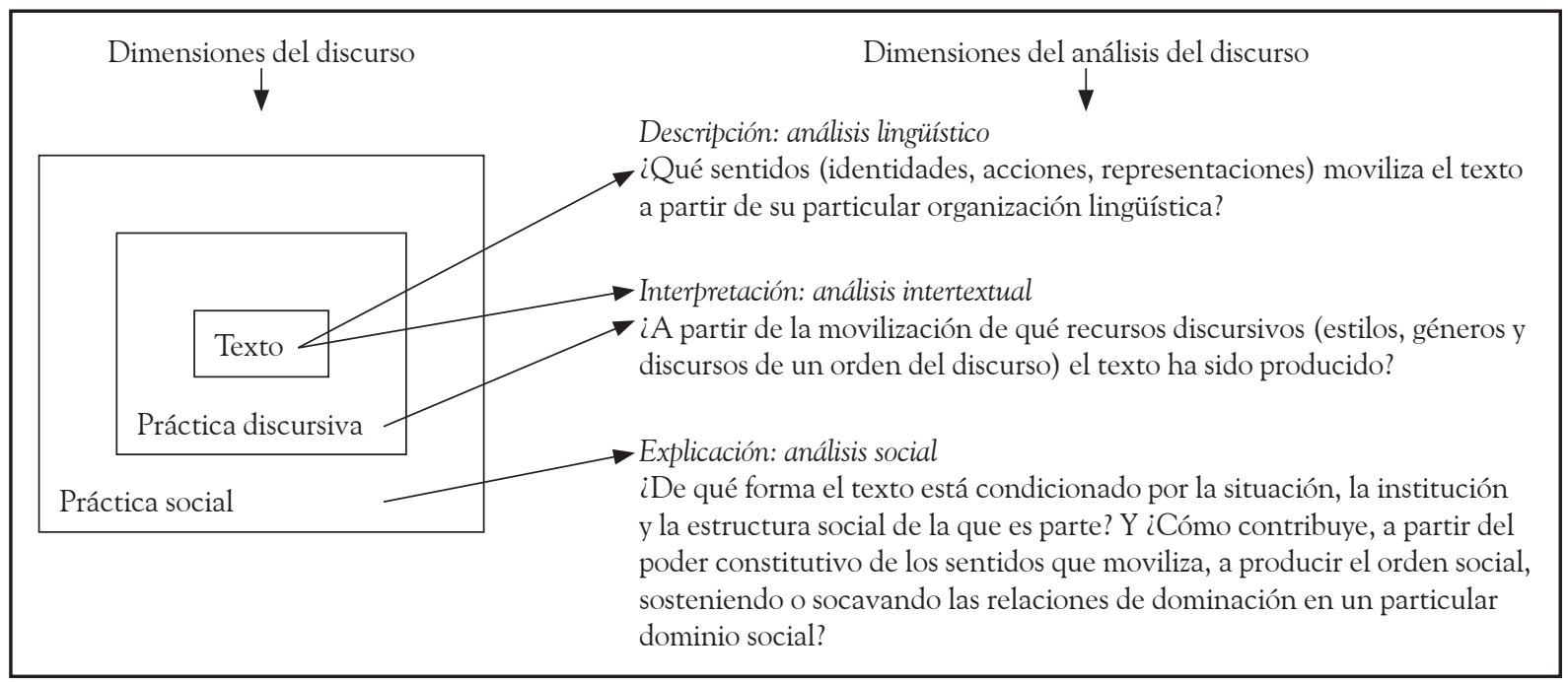

Figura 1

Modelo tridimensional del discurso.

Fuente: Fairclough, 1989. 
instituciones públicas, etc. y que circulan desde o hacia los nuevos espacios del trabajo? ¿De qué manera las nuevas prácticas discursivas que participan de la transformación de las organizaciones y de la experiencia de trabajo se tornan hegemónicas, invisibilizando y deslegitimando formas alternativas de representación, acción e identificación laboral, y contribuyendo a sostener las renovadas formas de dominación del capital sobre el trabajo? ¿Cuál ha sido el lugar del discurso psicológico en estos procesos de transformación sociolaboral? El objetivo de este artículo, más que responder dichas interrogantes, ha sido visibilizar el modo en que el ACD en general, y el modelo de Fairclough en particular, permiten formular este tipo de preguntas, ofreciéndonos un marco teórico-metodológico a partir del cual es posible explorar críticamente éstos y otros aspectos relativos a las dimensiones discursivas de las actuales transformaciones (por cierto múltiples, heterogéneas y contradictorias) del mundo del trabajo en América Latina.

\section{Consideraciones finales}

En este artículo hemos dado cuenta del desarrollo y consolidación en la última década de nuevos núcleos de investigación psicosocial sobre el trabajo en América Latina. Estos desarrollos, si bien heterogéneos y dispersos, comparten un conjunto de lineamientos que permiten hablar de ciertas tendencias de renovación en dicho campo, las que siguen siendo por cierto marginales en relación con las tradiciones hegemónicas, de corte comportamentalista y (neo)positivista, de la psicología del trabajo y las organizaciones. Hemos señalado, a su vez, que salvo algunas excepciones, estas nuevas producciones no han hecho un uso sistemático de las herramientas conceptuales y analíticas que ofrece el campo contemporáneo de los estudios del discurso, lo que constituye una debilidad no menor a la luz de la importancia de las dimensiones discursivas en los recientes procesos de cambio sociolaboral. En ese marco, el artículo ha presentado la perspectiva del ACD, y dentro de ésta, el modelo tridimensional de discurso desarrollado por Nor- man Fairclough, buscando destacar el modo en que dichas perspectivas pueden contribuir al estudio crítico del lugar del lenguaje en los procesos de flexibilización laboral y reestructuración productiva ocurridos en la región. Sobra decir que la apuesta de este artículo apunta, no al abandono de otras formas de investigación cuantitativa y cualitativa no discursivas, sino a un enriquecimiento de los estudios psicosociales del trabajo a partir de la incorporación de los recursos teóricos y metodológicos que ofrece el ACD, o más en general, el campo de los estudios contemporáneos del discurso.

Los argumentos acá presentados esperan contribuir al desarrollo, consolidación y articulación de los nuevos núcleos de investigación psicosocial sobre el trabajo en América Latina, los que constituyen una oportunidad para el fortalecimiento de una psicología sensible a los procesos históricos de cambio sociocultural, interesada en visibilizar y problematizar las antiguas y nuevas formas de dominación, y comprometida con la democratización de la sociedad.

\section{Referencias}

Antunes, R. (2001). ¿Adiós al trabajo? Ensayo sobre la metamorfosis y la centralidad del mundo del trabajo. San Pablo: Cortez Editora.

Adkins, L. \& Lury, C. (1999). The labour of identity: Performing identities, performing economies. Economy and Society, 2(4), 598-614.

Alvesson, M. \& Kärreman, D. (2000). Taking the linguistic turn in organizational research: Challenges, responses, consequences. The journal of applied behavioral science, 36 (2), 136-158.

Alvesson, M. \& Willmott, H. (2002). Identity regulation as organizational control: Producing the appropriate individual. Journal of Management Studies, 39(5), 619-644.

Antaki, Ch., Billig, M., Edwards, D. \& Potter, J. (2003). Análisis del Discurso implica analizar: crítica de seis atajos analíticos. Athenea Digital, 3, 14-35.

Baritz, H. (1961). Los servidores del poder: historia del uso de la ciencia social en la industria norteamericana. Madrid: Europa. 
Battistini, O. (Comp.). (2004). El trabajo frente al espejo. Continuidades y rupturas en los procesos de construcción identitaria de los trabajadores. Buenos Aires: Prometeo.

Bauman, Z. (2000). Trabajo, consumismo y nuevos pobres. Barcelona: Gedisa.

Berardi, L. (2003). Análisis Crítico del Discurso. Perspectivas Latinoamericanas. Santiago: Frasis.

Bolívar, A. (2004). Análisis Crítico del discurso de los académicos. Signos, 37(55), 7-18.

Boltanski, L. \& Chiapello, E. (2002). El nuevo espíritu del capitalismo. Madrid: Akal.

Bourdieu, P. (2000). Contrafuegos: reflexiones para servir a la resistencia contra la invasión neoliberal. Barcelona: Anagrama.

Braz de Aquino, C. \& Sandoval, J. (2002). Significados de la ciudadanía en las "sociedades no salariales". Una mirada desde América Latina. Cuaderno de Relaciones Laborales, 20(2), 411-432.

Calsamiglia, H. \& Tusón, A. (1999). Las cosas del decir. Manual de análisis del discurso. Barcelona: Ariel.

Codo, W. (2008). Salud mental y trabajo. México: Plaza y Valdez.

Coutinho, M. C., Krawulski, E. \& Soares, D. (2007). Identidade e trabalho na contemporaneidade: repensando articulações possíveis. Psicologia $\mathbb{E}$ Sociedade, 19, 29-37.

Chiapello, E. \& Fairclough, N. (2002). Understanding the new management ideology: A transdisciplinary contribution from critical discourse analysis and new sociology of capitalism. Discourse $\mathcal{E}$ Society, 13(2), 185-208.

Chouliaraki, L. \& Fairclough, N. (1999). Discourse in Late Modernity. Edinburgh: University Press.

Danzinger, K. (1996). The practice of psychological discourse. En C. Graumann \& K. Gergen (Eds.), Historical dimensions of psychological discourse (pp. 17-35). New York: Cambridge University Press.

De la Garza, E. (Coord.). (2000). Tratado latinoamericano de sociología del trabajo. México: F.C.E.

Díaz, X., Godoy, L., Stecher, A. \& Toro, J. P. (Coords.). (2006). Trabajo, Identidad y Vínculo Social. Reflexiones y experiencias en el capitalismo flexible. Santiago de Chile: UDP/Centro de Estudios de la Mujer.

Du Gay, P. (1996). Consumption and Identity at Work. Londres: Sage.
Fairclough, N. (1989). Language and power. Londres: Longman.

Fairclough, N. (1992). Discourse and Social Change. Cambridge: Polity Press.

Fairclough, N. (1995). Critical Discourse Analysis. The Critical Study of Language. Londres: Longman.

Fairclough, N. (2000a). Language and Neo-Liberalism. Discourse Eु Society, 11 (2), 147-148.

Fairclough, N. (2000b). Representaciones del cambio en el discurso neoliberal. Cuaderno de Relaciones Laborales, 16, 13-35.

Fairclough, N. (2001). The dialectic of discourse. Textus, 14(2), 231-242.

Fairclough, N. (2002). Language in New Capitalism. Discourse Eु Society, 13(2), 163-166.

Fairclough, N. (2003a). Analysing discourse. Textual analysis for social research. Londres: Routledge.

Fairclough, N. (2003b). El análisis crítico del discurso como método para la investigación en Ciencias Sociales. En R. Wodak \& M. Meyer (Eds.), Métodos de análisis crítico del discurso (pp. 179-203). Barcelona: Gedisa.

Fairclough, N. (2005). Peripheral Vision: Discourse analysis in organization studies. The case for critical realism. Organization Studies, 26(6), 915-939.

Fairclough, N. \& Wodak, R. (2000). Análisis Crítico del Discurso. En T. Van Dijk (Ed.), El discurso como interacción social (pp. 367-404). Barcelona: Gedisa.

Fowler, R., Hodge, B., Kress, G. \& Trew, T. (1979). Language and control. London: Routledge \& Kegan Paul.

García, C. \& Carvajal, L. (2007). Tecnologías empresariales del yo: la construcción de sujetos laborales en el contexto del trabajo inmaterial. Universitas Psychologica, 6(1), 49-58.

Gergen, K. (1996). Realidades y relaciones. Barcelona: Paidós.

Godoy, L. \& Stecher, A. (2008). La experiencia de mujeres asalariadas en Santiago de Chile: sentidos del trabajo e identidades de género. En B. G. Espinosa (Coord.), Mundos del trabajo: pluralidad y transformaciones contemporáneas (pp. 195-212). Quito: FLACSO.

Grant, D., Keenoy, T. \& Oswick, C. (1998). Discourse + Organization. London: Sage. 
Habermas, J. (1990). Conocimiento e interés. Madrid: Taurus.

Halliday, M. (1994). An Introduction to Functional Grammar. London: Edward Armold.

Hard, M. \& Negri, A. (2002). Imperio. Buenos Aires: Paidós.

Iñiguez, L. (Ed.). (2003). Análisis del Discurso. Manual para las Ciencias Sociales. Barcelona: UOC.

Jaworski , A. \& Coupland, N. (Eds.). (1999). The Discourse Reader. London: Routledge.

Knights, D. \& Morgan, G. (1991). Corporate strategy, organizations, and subjectivity: A critique. Organization Studies, 12(2), 251-273.

Leyva, G. (Ed.). (2005). La Teoría Crítica y las tareas actuales de la crítica. Barcelona: Anthropos.

Magalhaes, I. (2005). Introducao: A Análise de Discurso Crítica. DELTA, 21, 1-9.

Martín, L. (2001). New developments in discourse analysis: Discourse as social practice. Folia Linguistica, 35(1-2), 42-78.

Martín, L. \& Whittaker, R. (Eds.). (1998). Poder-Decir o el poder de los discursos. Madrid: Arrecife.

Mendes, A. M. (Org.). (2008). Trabalho e Saúde. O sujeito entre emancipacâo e servidâo. Curitiba: Jurua.

Morales, J. \& Cortes, M. T. (2005). Análisis crítico del discurso del proceso de paz (ACDP), entre el gobierno del Presidente Andrés Pastrana Arango y las FARC-EP. En L. Ramírez \& G. Acosta (Comp.), Estudios del discurso en Colombia (pp. 173-188). Medellín: Universidad de Medellín.

Mumby, D. \& Clair, R. (2000). El discurso en las organizaciones. En T. Van Dijk (Ed.), El discurso como interacción social (pp. 263-296). Barcelona: Gedisa.

Nardi, E. (2006). Etica, Trabalho e Subjetividade. Porto Alegra: UFRGS.

Pardo, M. L. \& Buscaglia, V. (2008). Pobreza y salud mental desde el Análisis Crítico del Discurso. Discurso y Sociedad, 2(2), 357-393.

Parker, I. (1992). Discourse dynamics: Critical analysis for social and individual psychology. London: Routledge.

Potter, J. \& Wetherell, M. (1987). Discourse and Social Psychology. London: Sage.

Pulido-Martínez, H. C. (2004). En busca de una psicología crítica en los ámbitos laborales. Universitas Psychologica, 3(2), 213-222.
Pulido-Martínez, H. C. (2007). Produciendo trabajadores modernos. Conocimiento psicológico y el mundo del trabajo en el sur. Universitas Psychologica, 6(1), 27-38.

Pulido, H. C. \& Carvajal, L. (2001). Discurso y construcción de identidad: el caso de los propietarios de vehículos del transporte urbano en Bogotá. Debates en Psicología, 4, 91-103.

Renteria, E. \& Malvezzi, S. (2008). Empleabilidad, cambios y exigencias psicosociales en el trabajo. Universitas Psychologica, 7(2), 319-334.

Román, J. A. (2008). Sujeto, trabajo y familia: compartiendo reflexiones y artefactos de investigación. En Á. Soto (Ed.), Flexibilidad laboral y subjetividades (pp. 253-277). Santiago: LOM.

Rorty, R. (1990). El giro lingüístico. Dificultades metafilosóficas de la filosofía lingüística. Barcelona: Paidós.

Rose, N. (1996). Inventing our selves: Psychology, power and personhood. New York: Cambridge University Press.

Sato, L. (2007). Processos cotidianos de organização do trabalho na feira libre. Psicologia $\mathbb{E}$ Sociedade, 19, 95-102.

Schvarstein, L. \& Leopold, L. (Eds.). (2005). Trabajo y subjetividad. Entre lo existente y lo necesario. Buenos Aires: Paidós.

Sennet, R. (2006). La cultura del nuevo capitalismo. Barcelona: Anagrama.

Sisto, V. (2005). Flexibilización laboral de la docencia universitaria y la gest(ac)ión de la Universidad sin Órganos: un análisis desde la subjetividad laboral del docente en condiciones de precariedad. En B. Levy \& P. Gentilli (Comp.), Espacio público y privatización del conocimiento. Estudios sobre políticas universitarias en América Latina (pp. 523-54). Buenos Aires: CLACSO.

Soto, A. (Ed.). (2008). Flexibilidad laboral y subjetividades. Santiago: LOM.

Stillerman, J. \& Winn, P. (2006). Introduction: Globalization and the Latin-American Workplace. International Labor and Working-Class History, $70(1), 1-10$

Taylor, S. (2001). Locating and conducting discourse analytic research. En M. Wetherell, S. Taylor \& S. Yates (Eds.), Discourse as Data (pp. 5-48). Londres: Sage. 
Thompson, J. B. (1984). Studies in the Theory of Ideology. Oxford: Blackwell.

Tittoni, J. (2007). Trabalho, Poder E Sujeicâo. Trajetórias entre o emprego, o desemprego e os "novos" modos de trabalhar. Porto Alegra: Dom Quixote Editora.

Todaro, R. \& Yáñez, S. (2004). El trabajo se transforma. Relaciones de producción y relaciones de género. Santiago: Centro de Estudios de la Mujer.

Van Dijk, T. (1993). Principles of critical discourse analysis. Discourse Eु Society, 4(2), 249-283.

Van Dijk, T. (2000). El discurso como interacción en la sociedad. En T. Van Dijk (Ed.), El discurso como interacción social (pp. 19-66). Barcelona: Gedisa.

Van Dijk, T. (2003a). Prólogo. En L. Iñiguez (Ed.), Análisis del Discurso. Manual para las Ciencias Sociales (pp. 11-16). Barcelona: UOC.

Van Dijk, T. (2003b). La multidisciplinaridad del Análisis Crítico del Discurso: un alegato a favor de la diversidad. En R. Wodak \& M. Meyer (Eds.), Métodos de análisis crítico del discurso (pp. 143-177). Barcelona: Gedisa.

Vargas, L., Aragón, D., Rivera, S. \& Trevisi, C. (2004). Discursos circulantes y construcción social de empresa en una organización del sector floricultor colombiano. Universitas Psychologica, 3(2), 197-212. Wheterell, M. (2001). Debates in Discourse Research. En M. Wetherell, S. Taylor \& S. Yates (Eds.), Discourse Theory and Practice. A reader (pp. 380-399). London: Sage.

Wodak, R. (2003). De qué trata el análisis crítico del discurso. Resumen de su historia, sus conceptos fundamentales y sus desarrollos. En R. Wodak \& M. Meyer (Eds.), Métodos de análisis crítico del discurso (pp. 17-34). Barcelona: Gedisa. 
